\title{
Is hugging a robot weird? Investigating the influence of robot appearance on perception of hugging
}

\author{
Gabriele Trovato, Member, IEEE, Martin Do, Ömer Terlemez, Christian Mandery, Hiroyuki Ishii, \\ Nadia Bianchi-Berthouze, Tamim Asfour, Member, IEEE, and Atsuo Takanishi, Member, IEEE
}

\begin{abstract}
Humanoid robots are expected to be able to communicate with humans using physical interaction, including hug, which is a common gesture of affection. In order to achieve that, physical embodiment has to be carefully planned, as a userfriendly design will facilitate interaction and minimise repulsion. In this paper, we investigate the effect of manipulating the visual/tactile appearance of a robot, covering wires and metallic parts with clothes, and the auditory effect too by enabling or disabling the connector of the hand. The experiment consists in a hugging interaction between the participants and the humanoid robot ARMAR-IIIb. Results after the participation of 24 subjects confirm the positive effect of using clothes to modify the appearance and the negative effect of noise, although not enough to completely relieve participants from the anxiety of a physical contact.
\end{abstract}

\section{INTRODUCTION}

Social robots, especially humanoids, are expected to perform human-like actions and adapt to human ways of communication in order to be part of the society. Amongst humans, physical interaction is also common in everyday communication for conveying either emotions or simple messages such as greetings. Therefore, physical embodiment of robots becomes crucial, as it has an effect on perception of social interactions [1].

Hug is a common gesture amongst humans, used to express empathy and affection, in which signals of friendliness or the absence of hostility are transmitted, and it is also used as a greeting [2]. A classification of greetings was attempted by Friedman [3], who put hug within a set of 12 basic greetings. Hug however is not common in certain cultures, such as Japanese, and very common in others, such as German: [4] features a comparative study between those two countries on the use of greeting words and gestures in different contexts.

Positive effects of hug on health have been reported by Elias et al. [5] (hug and hand-holding reduce the harmful physical effects of stress) and Light et al. [6] (more frequent partner hugs were linked to higher oxytocin levels, lower blood pressure, and lower heart rate in premenopausal women) among others. In these regards, robots offering a hug can potentially convey the same benefits.

\footnotetext{
*This work was supported by the "Strategic Young Researcher Overseas Visits Program for Accelerating Brain Circulation” program (頭脳循環を加 速する若手研究者 戦略的海外派遣プログラム) from the Japanese Society for the Promotion of Science.

G. Trovato is with the Graduate School of Advanced Science and Engineering, Waseda University, Tokyo, Japan; e-mail: contact@takanishi.mech.waseda.ac.jp.
}

Experiments in which participants hug a robot are not very common. Telenoid is a robotic system of telecommunication [7], which has been hugged by elderly subjects. Hugvie [8] is a device more specifically built for hugging. The user gives a hug while listening to a partner's voice, therefore feeling like he/she is hugging the partner and receiving affection. This experiment suggested that this kind of interaction may lead to a misattribution of behaviour due to hug. Several research efforts have been focused on haptics as well, such as [9], a mannequin torso which wears a jacket also worn by the user at the same time, and mimics the pressure from the user's hug, or the system for remote hugging in [10].

Regarding humanoid robots of human size, a greeting interaction experiment was performed in [11], where hug was one of the possible choices of greeting gesture. Robovie [12] interacted with children in supermarkets and asked them to hug, however the act of hugging was not the specific focus.

However, in the existing literature there are no studies that measure how it feels to hug a robot. This is a topic that spans from robot design and the Uncanny Valley to sociology and culture backgrounds. The user may feel comfortable after a hug, or be embarrassed to do it because of social conventions or cultural habits, or even afraid of the robot itself.

The acceptance of robots is related to the Uncanny Valley hypothesis and involves many aspects of robot design. A crucial aspect is the appearance: biology-inspired forms are documented in the design industry to be functional, pleasing to the eye and stimulating, thus details like soft curves and circles found in nature have appeal in the consumer product market [13]. Moreover, other aspects belonging to the auditory and kinesthetic fields, such as noise and vibration, are also important and should not be overlooked in robot design.

The robot we are using, ARMAR-IIIb [14], is composed of visible mechanical parts and visible cables; it also produces a noise caused by the cycling of the valves used for pneumatic actuation and makes the hand slightly vibrate.

Considering these concepts, our research question is to clarify what influence acceptance in a physical interaction such as a hug with a human-sized humanoid robot, varying two important aspects:

M.Do, Ö. Terlemez, C. Mandery and T. Asfour are with the Institute of Anthropomatics, Karlsruhe Institute of Technology, Karlsruhe, Germany.

H. Ishii is with the Research Institute for Science and Engineering, Waseda University, Tokyo, Japan.

N. Bianchi-Berthouze is with the University College London, UK.

A. Takanishi is with the Department of Modern Mechanical Engineering, Waseda University and with the Humanoid Robotics Institute (HRI), Waseda University, Japan. 
- the clothes, which have an impact on external appearance and on the sense of touch;

- the hand connector, which turns on/off hand vibration and noise, therefore influencing sense of touch and the auditory perception.

Our hypothesis is that hiding mechanical parts and wires, covering sharp edges and disabling the hand connector will have a positive influence on acceptance of the robot, and we measured it through questionnaires.

The rest of the paper is organized as follows: in Section II we describe the robot used for the experiment and in Section III the experimental setup; in Section IV we show the results, which are discussed in Section V; Section VI concludes the paper.

\section{HUMANOID ROBOT}

\section{A. Humanoid robot ARMAR-IIIb}

ARMAR-IIIb [14] is a humanoid robot with 43 degrees of freedom (DoF) designed for close cooperation with humans. It has a humanlike appearance in the upper body and sensory capabilities similar to humans. The robot has two anthropomorphic arms with 7 DoF each and an active head with 7 DoF which incorporates a visual and audio sensors such as a stereo camera system and several microphones. For dexterous grasping and manipulation, the robot is equipped with an anthropomorphic hand with 8 active DoF. The hand is driven pneumatically and incorporates a control system which allows the set the joint position and the air pressure of each joint by closing and opening the corresponding valves. Being highly compliant, and thanks to force/torque sensors, ARMAR-III can interact safely with other agents.

The hugging posture is shown in Fig.1: from that starting position, the robot slowly closes the arms to embrace the person, then releases. This hugging motion is created using the Master Motor Map (MMM) framework [15] [16], which aims to offer a unifying representation of motion for robotics applications, such as imitation learning, action recognition, motion analysis and synthesis, etc., and has already been used in several projects.

The representation of motion in the MMM framework is based on the MMM reference model, which provides a kinematic model with a total number of 104 degrees of freedom to describe whole-body motion. Additionally, the MMM provides converters to transfer motions of the MMM reference model to other kinematic embodiments like humanoid robots [15][17]. Since the kinematic of the humanoid robot differs from the reference model, it is not applicable to directly transfer joint angles from the reference model to it.

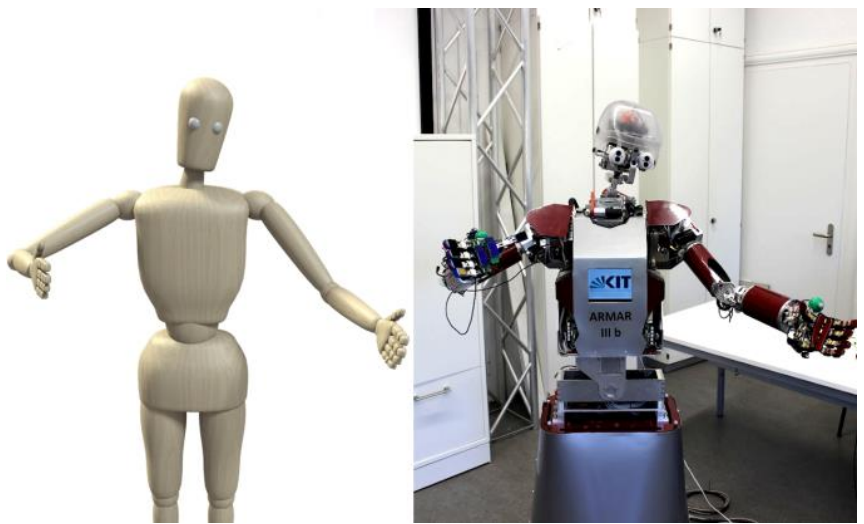

Figure 1. Implementation of the hugging initial posture: MMM model and final result

Instead, a step-wise non-linear optimization algorithm is used in which the pose of the humanoid robot is iteratively adjusted to select the pose featuring the highest similarity to the reference pose.

\section{B. Customisation of the robot}

ARMAR-IIIb is a robot designed with a pragmatic approach as defined by Hoffman [18]: "A pragmatic design approach sets out from specifications required of the robot's spatial activity towards physical goals [...]. Mechanical engineers design the robot's parts and relationships to fulfil these requirements [...] The resulting design is usually an assembly of limbs with more or less exposed links, actuators, and cables. [...] a shell is designed post-hoc to cover internal parts [...] The shape and structure of the shell is highly constrained by the existing core of the robot". This kind of robot design is sub-optimal for interaction and communication. This consideration is true not only for the exterior design but also for the sound and vibration produced by the hand. This fact is the reason why this robot was chosen for this experiment: by trying to compensate some of these lacks in design the robot's acceptance during physical interaction. In order to do so, we manipulated two variables:

- C 1/0: clothes on/off - the appearance of the robot is changed hiding where possible the cables, mechanical parts and sharp edges using some human clothes. In the clothes off condition, the head cap was removed in order to show the cables inside. The two appearances are shown in Fig.2. The presence of an apron is appropriate to the purpose of ARMAR, which usually performs grasping and manipulations in a kitchen environment. In this experiment too, it is placed inside a kitchen.

- H 1/0: hand on/off: the connector of the right hand (Fig. 3), which activates the hand control system, is enabled or disabled. It produces a noise caused by the cycling of the valves used for pneumatic actuation and makes the hand slightly vibrate. The noise can be perceived $65 \mathrm{~dB}$ loud at $50 \mathrm{~cm}$. The difference of the two conditions consists in sound and vibration, as the fingers do not move in any case. 


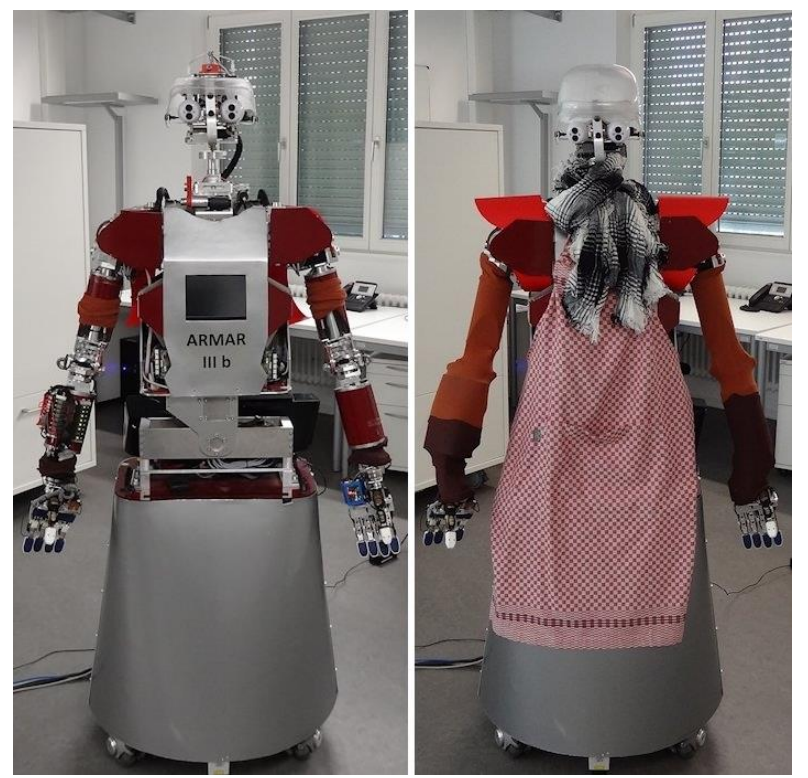

Figure 2. Two different appearances for ARMAR-IIIb: the usual one, with also the head cap removed (left) and the alternative one, wearing human clothes which hide wires and sharp edges (right).

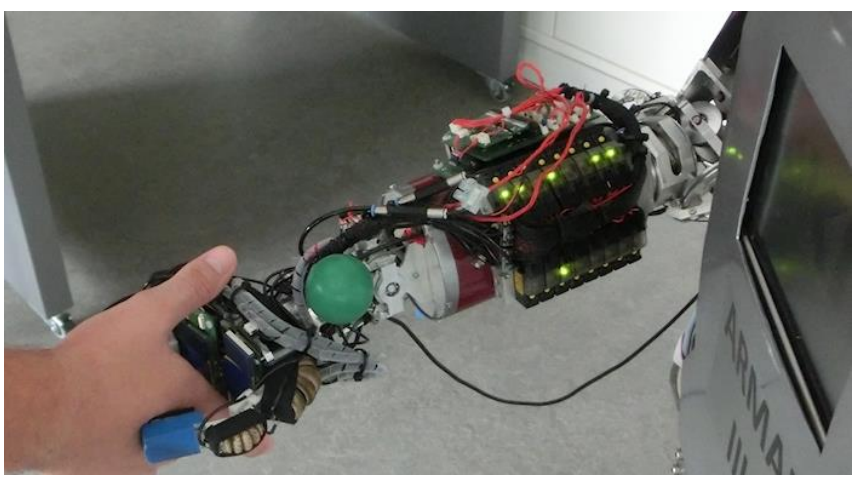

Figure 3. ARMAR's right hand when activated.

The two conditions are independent from each other, thus they are selected randomly for each participant's trial.

\section{EXPERIMENT SETUP}

\section{A. Recruitment policy}

In this experiment, hugging behaviour is intended to be a greeting. For this reason, as the experiment was run in Germany, only Germans or people living in Germany for a long while, accustomed to German way of greeting, were selected.

Another condition was that participants should not be roboticists, in order to avoid any bias in their opinion on what is uncanny in robot appearance; and should not know ARMAR-IIIb, otherwise they would know exactly its standard appearance.

\section{B. Room setup}

The room (Fig. 4) was arranged with a camera placed to one side of the robot, and tapes marking on the floor the distance from the participant's position to the robot's position in order to measure reaction times within proxemics. The lines on the floor indicate distance according to Hall's personal space [19], respectively 15, 46, 76, 122 and $210 \mathrm{~cm}$. Although data about proxemics has been collected, in this paper we specifically focus on the results of the questionnaires.

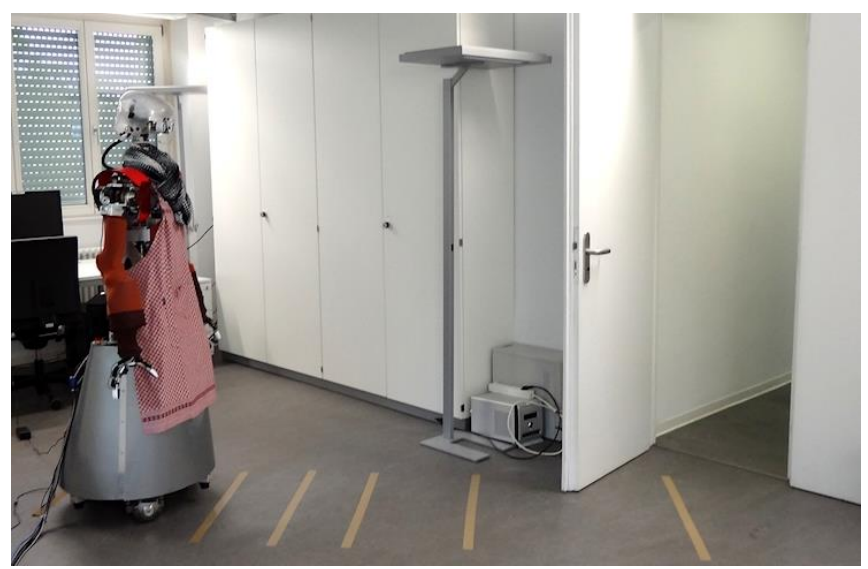

Figure 4. The room where the experiment was run, featuring ARMAR waiting standing 2 meters from the entrance.

\section{Protocol}

The experiment was composed of a dummy part (steps 03 ) and by the real hugging interaction (steps 4-7). The dummy part is necessary as introduction, because people don't hug someone who meet for the first time, but rather handshake. After getting more familiar, when meeting a 2nd time, a hug is more plausible.

The protocol is structured as in the following steps:

- $\quad$ Step 0: The robot is prepared according to randomly selected variables: dresses/undressed and hand cable connected/disconnected. The room is prepared as in Fig. 4.

- Step 1: The participant is instructed that the purpose of the experiment is to introduce our robot.

- Step 2: When the participant enters the room, the robot waiting about 2 meters distant gets triggered by an operator as the human participant approaches. The robot greets in German through a gesture and speech.

- Step 3: A short explanation about the robot follows, then the participant goes out of the room and fills a questionnaire regarding generic demographic questions as well as an evaluation of the greeting. This is the end of the dummy experiment.

- Step 4: The real experiment begins when the participant thinks that the experiment is over: as the participant enters the room again to hand over the questionnaire, the robot is triggered again and assumes a hugging posture with open arms. It does not approach the human.

- Step 5: If the participant does not spontaneously hug, he is verbally invited by the operator, who is present in the room, with an invite such as: "Don't you want to hug ARMAR?". This is done because in some cases 
the participant does not understand the robot's intention to hug. The participant is not forced in any way, and is free to refuse. When the person moves in the robot's arms, the operator will trigger another posture that make the arms tighter around the participant. Reaction time is measured related to personal distance markers.

- $\quad$ Step 6: The participant has to go to another room and fill a questionnaire (described in Section III.C) regarding the hug and the robot itself. In the meantime, robot's clothes and/or hand conditions are modified.

- $\quad$ Step 7: When the participant enters the room again to hand over the questionnaire, the robot wants to hug again. After the interaction, the participant has to indicate on the previous questionnaire whether something changed in the evaluation due to the new condition.

\section{Questionnaires}

Questionnaire featured 5-points semantic differential scales as well as open questions. It was structured in three parts:

The first was structured as follows.

$\begin{array}{rlllllll}\text { The act of hugging with ARMAR makes you feel } \\ \text { Anxious } & 1 & 2 & 3 & 4 & 5 & \text { Calm } \\ \text { Embarrassed } & 1 & 2 & 3 & 4 & 5 & \text { Not embarrassed } \\ \text { Uncomfortable } & 1 & 2 & 3 & 4 & 5 & \text { Comfortable } \\ \text { Scared } & 1 & 2 & 3 & 4 & 5 & \text { Fearless } \\ \begin{array}{r}\text { Concerned about } \\ \text { your safety }\end{array} & 1 & 2 & 3 & 4 & 5 & \text { Safe } \\ \begin{array}{r}\text { Other (please } \\ \text { specify) }\end{array} & & & & & \\ \end{array}$

The purpose is to clarify what the subject feels in hugging the robot, and possibly identify the reasons of discomfort.

The second part comprises the sets of scales "Anthropomorphism" and "Likeability" taken from the Godspeed series [20], and a similar measure called "Familiarity", which has been used in [21], and is made of the subscales Creepy/Pretty; Scary/Innocuous; Eerie/Familiar; Weird; Ordinary; Sinister/ Reassuring.

The third part was instead focused on specific aspects of ARMAR's appearance:

$\begin{array}{llllllll}\text { Do you like ARMAR's } & & & & & \\ \text { Face } & \text { Dislike } & 1 & 2 & 3 & 4 & 5 & \text { Like } \\ \text { Body } & \text { Dislike } & 1 & 2 & 3 & 4 & 5 & \text { Like } \\ \text { Size } & \text { Dislike } & 1 & 2 & 3 & 4 & 5 & \text { Like } \\ \text { Movement } & \text { Dislike } & 1 & 2 & 3 & 4 & 5 & \text { Like } \\ \text { Sound } & \text { Dislike } & 1 & 2 & 3 & 4 & 5 & \text { Like } \\ \text { Surface } & \text { Dislike } & 1 & 2 & 3 & 4 & 5 & \text { Like }\end{array}$

An additional space was left at the end for further comments.

\section{RESULTS}

\section{A. Demographics}

The experiment was run with the participation of 25 subjects. One had to be discarded as he was a roboticist who knew the robot already. The resulting group of 24 people was composed by 20 male and 4 female; age mean was 22.38 (SD 3.06). All subjects were either German or living in Germany for a long while.

All the participants interacted twice with the robot according to randomly selected conditions of the clothes $(\mathrm{C})$ and of the hand $(\mathrm{H})$, among $\mathrm{COH} 0, \mathrm{C} O \mathrm{H} 1, \mathrm{C} 1 \mathrm{H} 0$ and $\mathrm{C} 1 \mathrm{H} 1$. Some of the participants volunteered for a third trial and tried another condition, while some others refused to hug a second time.

\section{B. Evaluation}

In this section we report the findings obtained from the questionnaires, leaving detailed proximity analysis as future work.

\section{1) Habituation}

A preliminary statistical analysis was done to prove that there is no sign of habituation. Data was grouped by trial, and Wilcoxon Signed-Rank test was applied for each item of the questionnaire on 24 participants.

Effect of habituation was found only in the categories Anxious/Calm $(p=0.0156)$, and was not found in all the other items. We can conclude that the habituation effect is not relevant in this experiment. In any case, the order of the trials was randomised for each subject, and this will nullify the potential effect of habituation. The Anxious/Calm item was excluded from further analysis.

2) Reliability of the scales

The categories Anthropomorphism, Likeability and Familiarity are composed by 5 scales each. Their Cronbach's Alpha is respectively $0.82 ; 0.88$ and 0.79 . As shown by this result, we can consider the categories reliable.

Additionally, it is possible to calculate the Cronbach's Alpha of the first five scales described in Section III.D, which are not meant to be necessarily together, but are related each other as measurements of the subject's state. The result is 0.81 , meaning that these scales can also be considered as a single entity.

\section{3) Analysis of correlations}

Data was grouped by subjects who experienced variations of a variable (e.g. all the subjects who experienced $\mathrm{C} 0$ in the first trial and $\mathrm{C} 1$ in the second, or vice versa). In this way, the isolated effect of one variable gets highlighted. Wilcoxon Signed-Rank test was used.

Table I reports the results of this test. A significantly low $p$ value was found for Anthropomorphism, Likeability and Familiarity; in all the other scales, the small quantity of data prevents from finding relevant data; however, aggregating the scales about the subject's state in one category, a significant $p$ value was found for $\mathrm{C} 0 \rightarrow 1$. Nevertheless, the categories Sound and Surface showed respectively highly significant and marginally significant differences despite the low quantity of 
data. In all these results, the effect of $\mathrm{C} 0 \rightarrow 1$ is positive, while for $\mathrm{H} 0 \rightarrow 1$ it is negative.

TABLE I. DIFFERENCES OF THE TRIALS WITH DIFFERENT CONDITIONS, AND STATISTIC RELEVANCE

\begin{tabular}{|c|c|c|c|c|}
\hline \multirow{2}{*}{ Item } & \multicolumn{4}{|c|}{ Analysis } \\
\hline & $\mathrm{CO} \rightarrow \mathrm{I}$ & Wilcoxon & $H O \rightarrow 1$ & Wilcoxon \\
\hline $\begin{array}{l}\text { Embarassed/ } \\
\text { Not embarassed }\end{array}$ & +0.29 & $n=4$ & -0.08 & $n=4$ \\
\hline $\begin{array}{l}\text { Uncomfortable/ } \\
\text { Comfortable }\end{array}$ & +0.12 & $\begin{aligned} n & =6 \\
W & =-7\end{aligned}$ & -0.08 & $\begin{array}{l}n=10 \\
W=11\end{array}$ \\
\hline $\begin{array}{l}\text { Scared/ } \\
\text { Fearless }\end{array}$ & +0.24 & $n=3$ & -0.04 & $\begin{array}{l}n=6 \\
W=3\end{array}$ \\
\hline $\begin{array}{l}\text { Concerned } \\
\text { about safety/ } \\
\text { Safe }\end{array}$ & +0.18 & $n=3$ & -0.08 & $\begin{array}{l}n=8 \\
W=9\end{array}$ \\
\hline Subject's state & +0.19 & $\begin{array}{c}n=22 \\
W=-153 \\
p=\mathbf{0 . 0 1 3} * *\end{array}$ & -0.09 & $\begin{array}{c}n=29 \\
W=137\end{array}$ \\
\hline $\begin{array}{l}\text { Anthropomorp } \\
\text { hism }\end{array}$ & +0.2 & $\begin{array}{c}n=21 \\
W=-187 \\
p=\mathbf{0 . 0 0 1 2}^{* *}\end{array}$ & -0.24 & $\begin{array}{c}n=37 \\
W=463 \\
p=\mathbf{0 . 0 0 0 5}^{* * * *}\end{array}$ \\
\hline Likeability & +0.14 & $\begin{array}{c}n=14 \\
W=-90 \\
p=\mathbf{0 . 0 0 5}^{* *}\end{array}$ & -0.09 & $\begin{array}{c}n=18 \\
W=99 \\
\boldsymbol{p}=\mathbf{0 . 0 3 2} *\end{array}$ \\
\hline Familiarity & +0.31 & $\begin{array}{c}n=23 \\
W=-256 \\
p=\mathbf{0 . 0 0 0 1} * * *\end{array}$ & -0.21 & $\begin{array}{c}n=32 \\
W=354 \\
p=\mathbf{0 . 0 0 0 5}^{* * * *}\end{array}$ \\
\hline Face & +0.06 & $n=1$ & -0.04 & $n=1$ \\
\hline Body & +0.35 & $\begin{array}{c}n=7 \\
W=-18\end{array}$ & 0 & $\begin{array}{l}n=5 \\
W=0\end{array}$ \\
\hline Size & 0 & $n=2$ & 0 & $n=0$ \\
\hline Movement & 0 & $n=0$ & -0.04 & $n=1$ \\
\hline Sound & +0.18 & $\begin{aligned} n & =6 \\
W & =-6\end{aligned}$ & -1 & $\begin{array}{c}n=13 \\
W=91 \\
p=\mathbf{0 . 0 0 1 6}^{* *}\end{array}$ \\
\hline Surface & +0.53 & $\begin{array}{c}n=5 \\
\mathrm{~W}=-17\end{array}$ & 0 & $n=3$ \\
\hline
\end{tabular}

\subsubsection{Correlations}

By examining the correlations between the items in the questionnaire, we want to find if any of the ratings on ARMAR-IIIb's specific appearance are related (and possibly a causal factor) to the final impression and subject's state. Results are shown in Table II, where the analysis has been done through the calculation of Spearman's Rho. The value $\mathrm{R}$ indicates the type of correlation ( 1 if perfectly positive; 0 if perfectly negative).

Many correlations were found between the evaluation of head, body, and surface with many of the other questionnaire items.
TABLE II. CORRELATIONS BETWEEN SPECIFIC RATINGS OF ARMARIIIB'S APPEARANCE AND THE OTHER ITEMS

\begin{tabular}{|c|c|c|c|}
\hline \multirow[b]{2}{*}{ Item } & \multicolumn{3}{|c|}{ ARMAR-IIIb's appearance } \\
\hline & Face & Body & Size \\
\hline $\begin{array}{l}\text { Embarassed/ } \\
\text { Not embarassed }\end{array}$ & $\begin{array}{l}R=-0.11 \\
p=0.421\end{array}$ & $\begin{array}{l}R=0.266 \\
p=0.328\end{array}$ & $\begin{array}{l}R=-0.23 \\
p=0.081\end{array}$ \\
\hline $\begin{array}{l}\text { Uncomfortable/ } \\
\text { Comfortable }\end{array}$ & $\begin{array}{l}R=0.156 \\
p=0.246\end{array}$ & $\begin{array}{l}R=0.434 \\
p<0.001 * * *\end{array}$ & $\begin{array}{l}R=0.19 \\
p=0.158\end{array}$ \\
\hline $\begin{array}{l}\text { Scared/ } \\
\text { Fearless }\end{array}$ & $\begin{array}{l}R=0.289 \\
p=0.029 *\end{array}$ & $\begin{array}{l}R=0.281 \\
p=0.034^{*}\end{array}$ & $\begin{array}{l}R=0.037 \\
p=0.785\end{array}$ \\
\hline $\begin{array}{l}\begin{array}{l}\text { Concerned about } \\
\text { safety/Safe }\end{array} \\
\end{array}$ & $\begin{array}{l}R=0.415 \\
p=0.0013 * *\end{array}$ & $\begin{array}{l}R=0.242 \\
p=0.07\end{array}$ & $\begin{array}{l}R=0.019 \\
p=0.889\end{array}$ \\
\hline Anthropomorphism & $\begin{array}{l}R=0.262 \\
p=0.049 *\end{array}$ & $\begin{array}{l}R=0.493 \\
p<0.001 * * *\end{array}$ & $\begin{array}{l}R=0.391 \\
p=0.003 * *\end{array}$ \\
\hline Likeability & $\begin{array}{l}R=0.39 \\
p=0.003 * *\end{array}$ & $\begin{array}{l}R=0.386 \\
p=0.003^{* *}\end{array}$ & $\begin{array}{l}R=0.113 \\
p=0.404\end{array}$ \\
\hline \multirow[t]{2}{*}{ Familiarity } & $\begin{array}{l}R=0.249 \\
p=0.062\end{array}$ & $\begin{array}{l}R=0.398 \\
p=0.002 * *\end{array}$ & $\begin{aligned} R & =-0.071 \\
p & =0.599\end{aligned}$ \\
\hline & Movement & Sound & Surface \\
\hline $\begin{array}{l}\text { Embarassed/ } \\
\text { Not embarassed }\end{array}$ & $\begin{array}{l}R=0.071 \\
p=0.6\end{array}$ & $\begin{array}{l}R=-0.217 \\
p=0.105\end{array}$ & $\begin{array}{l}R=0.321 \\
p=0.014 *\end{array}$ \\
\hline $\begin{array}{l}\text { Uncomfortable/ } \\
\text { Comfortable }\end{array}$ & $\begin{array}{l}R=0.135 \\
p=0.318\end{array}$ & $\begin{array}{l}R=-0.08 \\
p=0.552\end{array}$ & $\begin{array}{l}R=0.195 \\
p=0.1464\end{array}$ \\
\hline $\begin{array}{l}\text { Scared/ } \\
\text { Fearless }\end{array}$ & $\begin{array}{l}R=-0.039 \\
p=0.774\end{array}$ & $\begin{array}{l}R=0.141 \\
p=0.296\end{array}$ & $\begin{array}{l}R=0.328 \\
p=0.013 *\end{array}$ \\
\hline $\begin{array}{l}\text { Concerned about } \\
\text { safety/Safe }\end{array}$ & $\begin{array}{l}R=0.036 \\
p=0.785\end{array}$ & $\begin{array}{l}R=0.165 \\
p=0.221\end{array}$ & $\begin{array}{l}R=0.287 \\
p=0.031 *\end{array}$ \\
\hline Anthropomorphism & $\begin{array}{l}R=0.568 \\
p<0.001 * * *\end{array}$ & $\begin{array}{l}R=0.068 \\
p=0.613\end{array}$ & $\begin{array}{l}R=0.411 \\
p=0.0015 * *\end{array}$ \\
\hline Likeability & $\begin{array}{l}R=0.026 \\
p=0.847\end{array}$ & $\begin{array}{l}R=0.137 \\
p=0.308\end{array}$ & $\begin{array}{l}R=0.382 \\
p=0.0034^{*} *\end{array}$ \\
\hline Familiarity & $\begin{array}{l}R=0.226 \\
p=0.091\end{array}$ & $\begin{array}{l}R=0.389 \\
p=0.003 * *\end{array}$ & $\begin{array}{l}R=0.331 \\
p=0.012 *\end{array}$ \\
\hline
\end{tabular}

\section{DISCUSSION}

In this section we summarise and discuss the relevant results found so far. We detected a significant difference in the categories Anthropomorphism, Likeability and Familiarity for both variables, suggesting a positive effect of clothes on conditions and a negative effect of hand on condition.

Evaluation of face, body and surface did not change across conditions except for the helmet and the clothes, however a positive correlation was found between them and almost all questionnaire items.

One limitation about the study is the a predominantly male sample group: this may limit the generalisability, because according to [22] females hug more often than male, however it does not invalidate the findings related to robot's appearance.

Regarding the interaction itself, limitations of this experiment can be summarised in two concepts: 1 . the timing and movement of the embracing behaviour of the robot have to be improved to be more natural (for example, learning from human motion capture data, using either the mapping 
approach described in [15] or by learning a DMP to learn a whole-body hugging primitive in task-space from motion capture); 2. improving robot appearance is not enough in some cases to relieve from anxiety of the act of hugging a robot, which is quite subjective. Analysis of proxemics of this experiment will provide additional details which should confirm or complement the present findings.

\section{CONCLUSION}

In this paper, we presented a study of human-robot physical interaction intended to provide findings on robot design. The experiment consisted in having participants hug the humanoid robot ARMAR-IIIb manipulating one variable regarding appearance, with or without clothes covering wires and metallic parts, and one variable about the auditory field, disabling or enabling the control of the hand, which generates a noise. Results highlighted the positive effect of using clothes to modify the appearance and the negative effect of noise. Given the participants behaviour, we can conclude that other aspects not examined in this study still concur to the feeling of anxiety in hugging interaction; however, the present findings also stressed out the importance of the auditory perception and the need for user-friendly design for robots that have to be used in close interaction. Future works include the repetition of the experiment with different kinds of humanoids including a comparison with androids, whose appearance is different enough to imply different uncanny perception, different expectation of robot intelligence, and different sense of touch during hugging interaction. Additional investigation will be also carried out on analysing the sound in order to determine what causes an uncanny effect, and to find an appropriate filter that nullifies it.

\section{ACKNOWLEDGEMENT}

The experiment was carried out in Karlsruhe Institute of Technology, Germany, thanks to InterACT, the Waseda/KIT exchange network. We thank all staff and students involved in the experiment.

\section{REFERENCES}

[1] J. Wainer, D. J. Feil-Seifer, D. A. Shell, and M. J. Mataric, "The role of physical embodiment in human-robot interaction," in The 15th IEEE International Symposium on Robot and Human Interactive Communication, 2006. ROMAN 2006, 2006, pp. 117-122.

[2] D. Morris, Bodytalk: a world guide to gestures. Jonathan Cape, 1994.

[3] H. S. Friedman, R. E. Riggio, and M. R. Di Matteo, "A classification of nonverbal greetings for use in studying face-to-face interaction," JSAS Catalog of Selected Documents in Psychology, vol. 11, pp. 31-32, 1981.

[4] S. Sugito, Aisatsu no kotoba to miburi (あいさつの言葉と身振り, "Words and gestures of greetings"). Bunkachou, 1981.

[5] M. Elias: "Study: hugs warm the heart, and may protect it". USA Today, 2003. Retrieved from http://www.usatoday.com/ news/health/2003-03-09-hug-usat_x.htm, March 10.

[6] K. C. Light, K. M. Grewen, and K. A. Amico: "More frequent partner hugs are linked to higher oxytocin levels, lower blood pressure, and heart rate in premenopausal women", Biological Psychology, 69, pp. 5$21,2005$.
[7] K. Ogawa, S. Nishio, K. Koda, K. Taura, T. Minato, C. T. Ishii, and H. Ishiguro, "Telenoid: Tele-presence Android for Communication," in ACM SIGGRAPH 2011 Emerging Technologies, New York, NY, USA, 2011, pp. 15:1-15:1.

[8] K. Kuwamura, K. Sakai, T. Minato, S. Nishio, and H. Ishiguro, "Hugvie: Communication Device for Encouraging Good Relationship through the Act of Hugging," Lovotics, vol. 1, no. 1, p. 10000104, 20014.

[9] N. Takahashi, R. Okazaki, H. Okabe, H. Yoshikawa, M. Yokoyama, and H. Kajimoto, "Sense-Roid: Emotional Haptic Communication with Yourself"," in Proceedings of Virtual Reality International Conference, France, 2011.

[10] J. K. S. Teh, A. D. Cheok, R. L. Peiris, Y. Choi, V. Thuong, and S. Lai, "Huggy Pajama: A Mobile Parent and Child Hugging Communication System," in Proceedings of the 7th International Conference on Interaction Design and Children, New York, NY, USA, 2008, pp. 250257.

[11] G. Trovato, M. Zecca, M. Do, Ö. Terlemez, M. Kuramochi, A. Waibel, and A. Takanishi, "A Novel Greeting Selection System for a CultureAdaptive Humanoid Robot," International Journal of Advanced Robotic Systems, vol. 12, no. 34, 2015.

[12] P. H. Kahn, T. Kanda, H. Ishiguro, N. G. Freier, R. L. Severson, B. T. Gill, J. H. Ruckert, and S. Shen, “'Robovie, you'll have to go into the closet now': children's social and moral relationships with a humanoid robot," Dev Psychol, vol. 48, no. 2, pp. 303-314, Mar. 2012.

[13] B. H. Schmitt and A. Simonson, Marketing Aesthetics. New York: Free Press, 2009.

[14] T. Asfour, K. Regenstein, P. Azad, J. Schroder, A. Bierbaum, N. Vahrenkamp, and R. Dillmann, "ARMAR-III: An Integrated Humanoid Platform for Sensory-Motor Control," in 2006 6th IEEE-RAS International Conference on Humanoid Robots, 2006, pp. 169-175.

[15] O. Terlemez, S. Ulbrich, C. Mandery, M. Do, N. Vahrenkamp and T. Asfour, Master Motor Map (MMM) - Framework and Toolkit for Capturing, Representing, and Reproducing Human Motion on Humanoid Robots, IEEE/RAS International Conference on Humanoid Robots (Humanoids), pp. 894 - 901, 2014

[16] P. Azad, T. Asfour, and R. Dillmann, "Toward an Unified Representation for Imitation of Human Motion on Humanoids," in 2007 IEEE International Conference on Robotics and Automation, 2007, pp. 2558-2563.

[17] M. Do, P. Azad, T. Asfour, and R. Dillmann, "Imitation of human motion on a humanoid robot using non-linear optimization," in 8th IEEE-RAS International Conference on Humanoid Robots, 2008, pp. $545-552$.

[18] G. Hoffman and W. Ju, "Designing Robots With Movement in Mind," Journal of Human-Robot Interaction, vol. 3, no. 1, pp. 89-122, Mar. 2014.

[19] E. T. Hall, The Hidden Dimension. Doubleday, 1966.

[20] C. Bartneck, D. Kulić, E. Croft, and S. Zoghbi, "Measurement Instruments for the Anthropomorphism, Animacy, Likeability, Perceived Intelligence, and Perceived Safety of Robots," Int J of Soc Robotics, vol. 1, no. 1, pp. 71-81, Jan. 2009.

[21] G. Trovato, J. G. Ramos, H. Azevedo, A. Moroni, S. Magossi, H. Ishii, R. Simmons, and A. Takanishi, “'Olá, my name is Ana': a study on Brazilians interacting with a receptionist robot," presented at the The 17th International Conference on Advanced Robotics, Istanbul, Turkey, 2015.

[22] V. J. Derlega, R. J. Lewis, S. Harrison, B. A. Winstead, and R. Constanza, "Gender difference in the initiation of intimacy", Journal of Nonverbal Behavior, 2, 83-96, 1989 